EConomía TeOría y PrÁctica • Nueva Época, número 47, julio-diciembre 2017, pp. 217-248, http://dx.doi.org/10.24275/ETYPUAM/NE/462017/Caligaris

\title{
Capital y renta diferencial de tipo II: una revisión crítica de la historia de las interpretaciones marxistas*
}

\section{Capital and Differential Rent II: a Critical Review of the History of Marxist Interpretations}

\author{
Gastón Caligaris** \\ Nicolás Pérez Trento***
}

\section{RESUMEN}

En este trabajo se realiza una reconstrucción crítica de la historia de las interpretaciones de la explicación marxiana de la renta diferencial de tipo II. En ella se muestra que la interpretación actualmente hegemónica dentro de la teoría marxista, en contraposición a como se la suele presentar, está lejos de ser única e incontrovertible. A su lado se encuentran al menos dos interpretaciones relevantes, una de las cuales ha imperado desde las primeras lecturas de la explicación marxiana hasta fines de la década de 1970. En el curso de esta reconstrucción se presentan cada una de estas interpretaciones, el contexto histórico en el que surgieron, su difusión y los debates que han suscitado. En el caso de la interpretación actualmente imperante se analiza en particular la evidencia textual que la misma presenta como justificación de su filiación marxiana. Finalmente, se realiza un balance crítico de las distintas interpretaciones.

Palabras clave: Renta de la tierra, renta diferencial de tipo II, teoría marxista, capital agrario, propiedad de la tierra.

Clasificación JEL: B140

\begin{abstract}
This paper presents a critical reconstruction of the history of interpretations of the Marxist explanation of differential rent II. We show that, in contrast to how it is commonly portrayed, the current hegemonic interpretation within Marxist theory is far from immutable and incontrovertible. Alongside it, there exist at least two relevant interpretations, one of which held sway from the first readings of Marx's explanation through the late nineteen-seventies. Each of these interpretations, as well as the historical context in which they emerged, their dissemination, and the debates they inspired, are synthetized in the course of this reconstruction. In the case of the interpretation currently in vogue, our analysis focuses in we particular on the textual evidence it cites to support its Marxist bona fides. Finally, we offer a critical assessment of the different interpretations.
\end{abstract}

Keywords: Ground Rent, Differential Rent Type II, Marxist Theory, Agrarian Capital, Ground Property. JEL Classification: B140

* Fecha de recepción: 05/10/2014. Fecha de aprobación: 09/02/2017.

**Universidad de Buenos Aires, Argentina. Correo electrónico: gcaligaris@gmail.com. ORCID 0000-0001-7672-2390

***Universidad de Buenos Aires, Argentina. Correo electrónico: nicolaspereztrento@ hotmail. com. ORCID 0000-0002-3520-3428 


\section{INTRODUCCIÓN}

Al igual que otras secciones de El Capital de Marx, la referida a la renta de la tierra ha sido históricamente objeto de diversas interpretaciones. En la actualidad, sin embargo, no pocas veces estas interpretaciones se presentan y se desarrollan de manera completamente autónoma, como si no existiesen alternativas, o quizás peor, se omite la referencia a sus autores originales y a los debates que han suscitado, presentando como novedosos argumentos que llevan varias décadas de existencia y que ya han sido sometidos a fuertes críticas. Dejando a un lado las omisiones deliberadas, generalmente la razón de esta especie de autismo teórico suele obedecer sencillamente al desconocimiento de la historia del pensamiento económico marxista, esto es, de la evolución de las diversas interpretaciones y controversias suscitadas. En particular, pensamos que en las últimas décadas ésta ha sido la situación para el caso de la renta diferencial de tipo II (RDII).

Como se advertirá tan pronto como nos introduzcamos en el análisis de los debates marxistas al respecto, la dilucidación de la naturaleza de este tipo de renta, lejos de presentarse como de una de esas "minucias y sutilezas" propias de la crítica marxiana de la economía política, se manifiesta como una tarea crucial para analizar la intensidad con que se invierte el capital en la producción agraria. En consecuencia, en un momento histórico como el actual, donde la producción agraria está siendo sometida a grandes cambios tecnológicos (Kloppenburg, 2004) y donde los pequeños capitales agrarios están siendo masivamente desplazados por capitales de tamaños nunca vistos en esta rama de la producción social (Bernstein, 2012), la necesidad de problematizar qué debe entenderse por la RDII se encuentra a la orden del día.

En este contexto, en el presente trabajo nos proponemos realizar una reconstrucción crítica de la historia de las diversas interpretaciones marxistas sobre este tipo de renta, con el doble propósito de llamar la atención sobre la existencia de interpretaciones alternativas y críticas a la concepción marxista actualmente dominante y de sentar las bases para un debate más amplio sobre las formas que adopta la inversión de capital en la producción agraria. Para ello, en la primera sección de este trabajo sintetizaremos algunas nociones básicas de la renta de la tierra que encuadran el debate. En las siguientes secciones presentaremos cada una de las interpretaciones identificadas, el contexto histórico en que surgen, su difusión y los debates que han suscitado. Luego, realizaremos un balance crítico de las diversas interpretaciones fundándonos en la evidencia textual que presenta cada una de ellas como sustento de su filiación marxiana. Finalmente, expondremos las principales conclusiones que se desprenden de esta investigación. 


\section{LA RENTA DE LA TIERRA EN LA CRÍTICA MARXIANA DE LA ECONOMÍA POLÍTICA}

Antes de examinar la historia de las diversas interpretaciones marxistas sobre la RDII conviene señalar algunos puntos básicos de la explicación marxiana de la renta de la tierra que subyacen toda interpretación. Ante todo, es relevante notar que el legado de Marx respecto a su explicación de la renta de la tierra es bastante acotado, al menos en relación con otras partes de su crítica de la economía política. De hecho, existen sólo dos textos, escritos ambos como borradores, en donde Marx alcanza a abordar la cuestión: el primero es su revisión crítica de la teoría de la renta de la tierra de Rodbertus y de la economía política clásica, escrita entre los años 1862-1863 (Marx, 1987 [1862-1863], pp. 7-341), y el segundo es el capítulo correspondiente a la renta de la tierra del único borrador existente de lo que luego se publicó como el tercer tomo de El Capital, escrito en 1865 (Marx, 1997 [1865], pp. 791-1034). Ocurre que, incluso en sus planes menos ambiciosos, la explicación de la renta de la tierra ocupaba un lugar muy avanzado en la estructura argumental de la crítica marxiana de la economía política. ${ }^{1}$ En efecto, para Marx la renta de la tierra específicamente capitalista, cualquiera haya sido su origen histórico, debía ser concebida ante todo como una forma concreta particular adoptada por el plusvalor y como tal sólo comprensible luego de haber explicado "sistemáticamente" las determinaciones generales de la producción y circulación del capital (Guidi, 1998). ${ }^{2}$ En suma, se puede concluir que, al igual que otras partes de la crítica marxiana de la economía política, la correspondiente a la

\footnotetext{
${ }^{1}$ Sobre el alcance y la realización de los diversos planes de exposición de la crítica marxiana se ha discutido extensamente en la literatura especializada (Grossmann, 1979 [1929]; Rosdolsky, 1989 [1968]; Rubel, 2003 [1973]; Fineschi, 2013, entre otros). En particular, uno de los temas en debate ha sido si el "libro sobre la propiedad de la tierra", planeado por Marx a fines de la década de 1850 (Marx, 1997 [1859], pág. 3), fue finalmente incorporado como una sección más de El Capital o quedó pendiente de realización (véase al respecto especialmente Arthur, 2006). En cualquier caso, nadie ha cuestionado el lugar avanzado que ocupa el tratamiento de la renta de la tierra en la exposición de la crítica marxiana.

${ }^{2}$ La cuestión del vínculo entre el "análisis histórico" y el "análisis sistemático" en la exposición marxiana de la crítica de la economía política ha sido particularmente debatido por los autores de la llamada "Neue-Marx Lekture" (Backhaus, 1978 [1969]; Reichelt, 1970; Schmidt, 1973 [1972]) y de la "New Dialectics" (Arthur C., 1997; Robles Baez, 1999; Reuten, "The Interconnection of Systematic Dialectics and Historical Materialism", 2000). En línea con estas interpretaciones, consideramos que la exposición marxiana es, pace Engels (1997 [1859]), esencialmente "sistemática". De ahí, que la renta de la tierra sólo pueda ser tratada con posterioridad al desarrollo de las determinaciones generales del capital, incluyendo al capital prestado a interés. Sin embargo, tal como se ha procurado demostrar en otro lugar, ello no implica que el "análisis histórico" no forme parte sustantiva del "método dialéctico" que guía la exposición marxiana (Caligaris y Starosta, 2017).
} 
renta de la tierra no alcanzó nunca el estatus de una exposición acabada y definitiva (Reuten, G., 2002 y Moseley, F., 2015).

Otro aspecto destacable de la explicación marxiana de la renta de la tierra y, como veremos, aún más presente en el trasfondo de las diversas interpretaciones marxistas de la RDII, es su vínculo con las explicaciones formuladas por la economía política clásica y en particular por Ricardo. En efecto, tal como ha sido reconocido por la literatura especializada, en la explicación marxiana de la renta de la tierra la impronta de las explicaciones precedentes del fenómeno, y especialmente de la ricardiana, es claramente manifiesta (Murray, 1977; Howard y King, 1988; Gehrke, 2012, entre otros). De hecho, en este sentido no parece casual que la primera de las únicas dos formulaciones sistemáticas de la concepción marxiana de la renta haya sido realizada en el contexto de la revisión crítica de las concepciones presentes en la economía política clásica. Más importante aún, del análisis de su correspondencia se puede inferir que el establecimiento de sus diferencias con la teoría ricardiana de la renta formaba parte de las principales preocupaciones que Marx tenía sobre la cuestión (véase especialmente, Marx (1983 [1845-1895], p. 122). Como veremos, en las lecturas clásicas de la explicación marxiana de la renta de la tierra, la diferencia con la explicación ricardiana pasaba exclusivamente por el reconocimiento de la llamada "renta absoluta" en oposición a la "renta diferencial" (véase, por ejemplo, Kautsky 2002 [1899]). Modernamente, en cambio, se reconoce que las diferencias entre ambas explicaciones son más profundas y remiten, en última instancia, a las diferentes concepciones que tenían Marx y Ricardo sobre el proceso de formación de los precios o, más profundamente aún, a sus respectivas teorías del valor (véase, por ejemplo, Murray, 1977 y Klimovsky, 1985). ${ }^{3}$ Más adelante, discutiremos hasta qué punto estás diferencias esenciales juegan un papel en las distintas interpretaciones existentes sobre la RDII.

Finalmente, vale la pena reponer sucintamente cuál es la concepción de la renta diferencial en general y de la RDII en particular que subyace a las distintas interpretaciones que examinaremos. De manera general, se puede decir que la valorización del capital a la tasa normal de ganancia emerge simplemente de la competencia entre los capitales individuales con base en el aumento de la productividad del

\footnotetext{
${ }^{3}$ Las diferentes concepciones que tenían Marx y Ricardo sobre la determinación de los precios $\mathrm{y}$, en última instancia, del valor de las mercancías ha sido una cuestión extensamente discutida en la literatura especializada. Dentro de la teoría marxista no existe actualmente un consenso general sobre la explicación marxiana de la determinación de los precios y, por lo tanto, de su diferencia con la explicación ricardiana. Para una síntesis de las distintas posiciones existentes en la teoría marxista contemporánea respecto de la explicación marxiana de la determinación de los precios véase Moseley (2016, p. 221 y ss.). Respecto a los debates actuales en torno a la teoría marxiana del valor véase Kicillof y Starosta (2007a; 2007b).
} 
trabajo. Sin embargo, en determinadas ramas de la producción, este mecanismo simple choca con la existencia de condiciones naturales específicas que afectan dicha productividad. Así, para tomar el ejemplo clásico de la producción agraria y en torno al cual se han estructurado la mayoría de los debates dentro de la literatura marxista, la existencia de tierras de mejor fertilidad y/o ubicación que otras dentro de las tierras disponibles para la producción, redunda en la existencia de diferentes productividades del trabajo y, en consecuencia, en distintas tasas de ganancia para una misma inversión de capital. En este contexto, dado que las plusganancias generadas por encima de la tasa normal de ganancia no corresponden al movimiento del capital, sino exclusivamente a las particularidades de cada parcela de tierra, las mismas se constituyen en rentas de la tierra. En la medida en que se trata de una renta que surge específicamente de la puesta en acción de una productividad del trabajo mayor a la que determina la valorización normal del capital, Marx la denomina, siguiendo la terminología propia de la economía política, renta diferencial de la tierra. En sus palabras,

la renta diferencial ... [es] el resultado de la productividad diferente de iguales inversiones de capital en iguales superficies de terreno de diferente fertilidad [y/o ubicación], de tal manera que la renta diferencial resulta ... determinada por la diferencia entre el rendimiento del capital invertido en el suelo peor ... y el del capital invertido en el suelo mejor (Marx, 1997 [1865], p. 865).

Dada esta situación, Marx considera el caso en donde "volúmenes de capital de diferente productividad [del trabajo] se inviertan sucesivamente en un mismo predio" (Marx, 1997 [1865], p. 865). El resultado también es el de la generación de una plusganancia que finalmente se convierte en renta diferencial de la tierra. Pero, en la medida en que surge bajo una forma concreta diferente a la renta diferencial desarrollada en primer lugar, Marx la especifica como RDII. Así, de manera simple, este tipo de renta diferencial queda definida no por la inversión de un mismo capital sobre distintos tipos de tierra, sino por la inversión de diversos montos de capital sobre un mismo tipo de tierra o, lo que es lo mismo, como la renta diferencial que surge de la posibilidad de ampliar la producción no mediante la ampliación extensiva de la magnitud de capital hacia otras tierras sino mediante su aplicación intensiva. Como veremos a continuación, esta definición simple de la RDII está lejos de agotar la complejidad de este tipo de renta. En efecto, es precisamente la cuestión de las formas concretas que toma esta inversión intensiva del capital la que da lugar a las distintas interpretaciones de la RDII. Pasemos, por consiguiente, a analizar estas distintas interpretaciones. 


\section{LA INTERPRETACIÓN CLÁSICA}

La primera interpretación de la RDII es la que desarrollan los primeros marxistas a pocos años de publicado el tercer volumen de El Capital, y que prevalecerá prácticamente sin variantes hasta mediados de la década de 1970. De acuerdo con esta interpretación, que aquí denominaremos "clásica", las referidas plusganancias que constituyen la RDII surgen de las inversiones sucesivas de cuotas de capital que se aplican sobre una misma parcela de tierra, siendo cada una de ellas portadora de una productividad del trabajo menor a la anterior, hasta el punto en que la última inversión alcanza la productividad del trabajo correspondiente a la que determina el precio de mercado, esto es, hasta el punto en que dicha última inversión no arroja más que la tasa normal de ganancia. Un breve y simple ejemplo numérico puede servir para ilustrar la esencia de esta interpretación.

Tabla $\left.\right|^{4}$

\begin{tabular}{|c|c|c|c|c|}
\hline CP & Q & Ppi & PPg & R \\
\hline 100 & 10 & 10 & 50 & 400 \\
\hline 100 & 5 & 20 & 50 & 150 \\
\hline 100 & 2 & 50 & 50 & 0 \\
\hline
\end{tabular}

Según esta interpretación, en vez de invertir un nuevo capital en una tierra de fertilidad o ubicación peor, la ampliación de la producción puede resolverse mediante la aplicación de una nueva cuota de capital que ponga en marcha un trabajo de menor productividad en una tierra ya en actividad. Así, siguiendo este ejemplo, se puede poner una segunda cuota de capital de $\$ 100$ cuya productividad del trabajo de $5 / 100$ sea menor a la ya existente de $10 / 100$, pero mayor a la que determina el precio de producción general de 2/100. De este modo, esa segunda cuota o inversión de capital aún podrá arrojar una plusganancia de $\$ 150$. Y aún se puede poner una tercera cuota de capital que iguale la productividad del trabajo que determina el precio de producción general, en cuyo caso sólo se obtendrá la tasa normal de ganancia.

Las primeras versiones de esta interpretación pueden encontrarse en las obras de Kautsky (2002 [1899], p. 79 y ss.) y Lenin (1981 [1901], p. 104 y ss.; 1983

${ }^{4}$ Donde CP es el costo de producción (el capital total adelantado más la ganancia media), Q es la cantidad de producto, PPI es el precio de producción individual, PPG es el precio de producción general y RDII es la renta de la tierra de tipo II. 
[1908], p. 290), ${ }^{5}$ y ya entrado el siglo Xx en las de autores como Lapidus y Ostrovitianov (1929, p. 276 y ss.) para el caso de la tradición soviética, y Mandel (1972 [1962], p. 254 y ss.) en la tradición trotskista, tan sólo para nombrar a los autores más populares. Para esta época, es notable que incluso los críticos de Marx se atenían también a esta interpretación de la RDII. Un caso notable en este sentido es el de Diehl (1899), quien desarrolla la que probablemente sea la lectura más pormenorizada y atenta de la explicación marxiana de la renta de la tierra durante el primer periodo de recepción del volumen tercero de El Capital (Diehl, 1899, p. 441 y ss.).

El caso de La cuestión agraria de Kautsky, por tomar quizás a la obra más célebre y de mayor difusión del primer período, es bastante ilustrativo de este tipo de interpretación:

Finalmente, he aquí ahora un tercer tipo de renta del suelo; [...] si puede aumentar la producción de los medios de subsistencia, poniendo en explotación no solamente una tierra aún no cultivada sino también mejorando la calidad de la tierra ya sometida a explotación, por medio de un mayor empleo de trabajo, por medio de una mayor inversión de capital [...]. Si este capital adicional, invertido en un terreno mejor, obtiene un provecho mayor del que se logra cultivando en el terreno peor -que de cualquier manera debe ser explotado- este mayor provecho constituye un nuevo superbeneficio, una nueva renta de la tierra [...] Supongamos que [...] se realiza en el lote A una inversión adicional de capital que duplique, por ejemplo, el capital inicial, y que la nueva inversión no sea de la misma productividad que el primero [de 420/3200 en vez de 450/3200], pero que sea más productivo que la inversión en el terreno peor [de 400/3200]. Tendremos entonces: [...] La renta de A ha aumentado, por lo que respecta a la masa total, sobre la inversión adicional A2 (Kautsky, 2002 [1899], p. 85).

Visto retrospectivamente, se trata de una interpretación bastante simple y rudimentaria, donde toda la riqueza de la exposición marxiana de este tipo de renta queda dejada de lado. En efecto, la explicación marxiana de la RDII no se diferenciaba cualitativamente, hasta aquí, de la presentada por Ricardo (1985 [1817], p. 54). Así, para la interpretación clásica, en este punto las diferencias con la explicación ricardiana se reducían simplemente a la mayor precisión con que Marx

${ }^{5}$ Otro de los primeros marxistas que se inscriben en esta línea, aunque con menor trascendencia teórica, es Julien Borchardt. El caso es relevante en cuanto la publicación de su Edición Popular de El Capital, donde presenta esta interpretación (Borchardt, 1981 [1919], p. 365), se convirtió rápidamente en uno de los resúmenes más célebres y populares de la obra de Marx entre los marxistas de la Segunda Internacional (Sweezy, 1973 [1942], p. 177 n.). Las numerosas traducciones que tuvo esta obra son un claro indicador de su difusión ulterior. 
presentaba las relaciones cuantitativas en que se expresa la RDII. Murray ofrece un ejemplo paradigmático de esta interpretación al afirmar que, en su explicación de la RDII, Marx "buscaba romper con la relación hermética que Ricardo había establecido entre el incremento del producto, la caída de la fertilidad y el crecimiento de la renta" (Murray, 1977, p. 104). En contraposición, durante todo el periodo en que domina la interpretación clásica, el foco de los debates en torno a la explicación marxiana de la renta, y en particular su diferencia esencial con la explicación ricardiana, está puesto en la consideración de la llamada por Marx "renta absoluta" (Kautsky, 2002 [1899]; Diehl, 1899; Lenin, 1981 [1901]; Bortkiewicz, (1979) [1910-11]; Emmanuel, 1972 [1969]; Vergopoulos, 1977 [1974]).

\section{LAS INTERPRETACIONES MODERNAS}

Hacia fines de la década de 1970 la interpretación clásica es desafiada por dos nuevas interpretaciones, una de las cuales rápidamente se volvió hegemónica. Este viraje en la historia de la teoría marxiana de la renta de la tierra no es casual. En primer lugar, se desarrolla en el marco de una renovación de las lecturas sobre la renta de la tierra motivada, de una parte, por la llamada "crisis del petróleo" de principios de la década del los 70 que había provocado la suba abrupta de los precios de las mercancías portadoras de renta de la tierra (Murray, 1977; Flichman et al., 1981, entre otros) y, de otra parte, por el desarrollo de movimientos políticos campesinos, en especial en América Latina, que habían vuelto a poner en la agenda de discusión la vieja cuestión de la subsistencia del pequeño campesino (Amin y Vergopoulos, 1977 [1974]; Gutelman, 1978 [1974]; Bartra, 1979 [1976], entre otros). En segundo lugar, estas nuevas interpretaciones se desarrollan en un contexto teórico dentro del marxismo dominado por las críticas a las llamadas lecturas ricardianas o "tecnológicas" de la teoría marxiana del valor (Backhaus, 1978 [1969]; Elson, 1979; De Vroey, 1982, entre otros), lo cual conducía a una revaluación de las diferencias entre la explicación ricardiana y marxiana en todos los niveles de la teoría económica.

\section{IV.1. La primera crítica a la interpretación clásica}

El primer intento de desafiar la hegemonía de la interpretación clásica es el de Michael Ball (1977). Según este autor, el principal defecto de las lecturas tradicionales de la teoría marxiana de la RDII es que pasan por alto la diferencia sustancial que existe entre la explicación marxiana y la ricardiana de dicho tipo de renta, diferencia que se remonta en última instancia a la divergente concepción del valor 
que subyace a ambas explicaciones. En efecto, de acuerdo con Ball, toda la cuestión pasa por la forma en que se calcula el valor y, en consecuencia, el precio de producción de la mercancía agraria.

De acuerdo con esta interpretación, el cálculo ricardiano del precio de producción de la mercancía agraria surge de tomar como referencia exclusivamente la última inversión de capital, esto es, aquella que porta la menor productividad del trabajo. Se trata, en efecto, del mismo procedimiento que realiza lo que aquí denominamos la interpretación clásica de la RDII. De acuerdo con el ejemplo que vimos en la sección anterior, el precio de mercado de las mercancías agrarias producidas en la parcela de tierra considerada sería de $\$ 50$, precisamente porque ése es el precio de producción individual que corresponde a la última cuota o inversión de capital. Este cálculo, sin embargo, es considerado por Ball como "marginalista, ya que considera cada inversión adicional de capital sobre una base incremental, en vez de tratar al total de capital invertido en cada parcela como una totalidad", lo cual resulta de "la incapacidad de Ricardo para salirse del chaleco de fuerza teórico de considerar sólo el tiempo de trabajo concreto" (Ball, 1977, p. 383, traducción propia). En consecuencia, de acuerdo con Ball, el cálculo verdaderamente consistente con la teoría marxiana del valor debe resultar de considerar al capital total invertido como una sola unidad. $\mathrm{O}$ bien, puesto en términos ricardianos, debe resultar de la consideración del promedio de los distintos precios de producción correspondientes a cada una de las inversiones de capital consideradas. Siguiendo con el ejemplo anterior, el capital total invertido más la ganancia media correspondiente sería de $\$ 300$ que, arrojando un producto total de 17, daría un precio unitario de $\$ 17,65$. Luego, la diferencia entre este precio y el precio de mercado daría como resultado la formación de la RDII. ${ }^{6}$

La diferencia entre ambos procedimientos puede apreciarse en las siguientes tablas:

${ }^{6}$ Es posible encontrar un antecedente de esta interpretación en la obra El capitalismo y la agricultura de Serguéi Bulgakov, aunque de acuerdo con la interpretación clásica de la teoría marxiana de la RDII, el error de cálculo era imputado indistintamente a Marx y a Ricardo. La formulación de esta interpretación fue recogida por Lenin en 1901: "La noción de la última y menos productiva inversión de trabajo y de capital" - prosigue objetando el señor Bulgakov-, "es utilizada sin crítica tanto por Ricardo como por Marx. No es difícil advertir el elemento de arbitrariedad introducido por esta noción. Supongamos que se invierte en la tierra un capital de $10 a$, y que cada $a$ sucesiva da una productividad menor; la producción total será $A$. Es evidente que el promedio de rendimiento de cada $a$ será igual a A/10, y si consideramos el capital como un todo, es precisamente este rendimiento medio el que determinará el precio" (Lenin, 1981 [1901], p. 122). 
Tabla I. Interpretación clásica

\begin{tabular}{|c|c|c|c|c|}
\hline$C P$ & Q & Ppi & PPg & $R$ \\
\hline 100 & 10 & 10 & 50 & 400 \\
\hline 100 & 5 & 20 & 50 & 150 \\
\hline 100 & 2 & 50 & 50 & 0 \\
\hline 300 & 17 & & & 550 \\
\hline
\end{tabular}

Tabla II. Interpretación de Ball

\begin{tabular}{|c|c|c|c|c|}
\hline$C P$ & $\mathrm{Q}$ & Ppi & PPg & $\mathrm{R}$ \\
\hline 300 & 17 & 17,65 & 50 & 550 \\
\hline
\end{tabular}

Hasta aquí la diferencia entre ambos cálculos parece del todo superflua. En efecto, como lo ha notado perspicazmente Barnes, tanto en uno como en otro, la masa de capital, de ganancia y de renta es exactamente la misma (Barnes, 1984, p. 130). Sin embargo, para Ball la diferencia es crucial en cuanto sólo en el cálculo marxista la renta aparece determinando el precio de mercado y, más importante aún, limitando la acumulación de capital, dos determinantes que la interpretación clásica trata como exclusivos de la renta absoluta. El argumento del autor es el siguiente. Si se considera el cálculo marxista del precio de producción, la inversión de capital en una misma parcela de tierra podría continuar hasta que el conjunto del capital alcance la ganancia normal. Esto es, siguiendo con el mismo ejemplo numérico, si al aplicarse la tercera inversión de capital el precio es de $\$ 17,65$, entonces se podrían seguir aplicando nuevas inversiones, cada una más improductiva que la anterior, hasta alcanzar el precio promedio que corresponde al precio de mercado de \$50. La existencia de la propiedad de la tierra, sin embargo, impide que esta inversión sucesiva se desarrolle, ya que sólo podría realizarse a expensas de la renta de la tierra. Esto es, si con la tercera inversión de capital se está cobrando una renta de $\$ 550$, una cuarta inversión con una productividad menor a la anterior, supongamos de 1/100 sólo podría aplicarse si el terrateniente cediese $\$ 50$ de su renta. Así, la existencia misma de la renta diferencial de la tierra se constituye en un determinante del nivel de precios y de la inversión de capital. En palabras del autor:

El hecho de que los arrendatarios sólo sean influenciados por el precio de producción promedio de cada suelo produce un resultado interesante. Si el capital se moviera libremente en la agricultura, la acumulación llevada adelante por los arrendatarios capitalistas resultaría en inversiones adicionales de capital sobre cada suelo hasta que los precios de producción se hubieran igualado en todas las tierras cultivadas [...]. La 
existencia de la propiedad privada actúa como una barrera a esta igualación de los precios de producción individuales. Una vez que la renta se ha pagado en las tierras con menores precios de producción, no habría razón para que el terrateniente consintiera perder la renta que ya está cobrando si el capitalista fuera a incrementar el producto mediante la inversión adicional de capital a expensas de la renta. [...] La teoría de la renta diferencial de Marx muestra que la renta afecta los precios, y limita la acumulación del capital. (Ball, 1977, pp. 390-391, traducción propia).

De las variadas críticas que ha recibido la interpretación de Ball, tal vez la más elaborada haya sido la de Evans (1992). Según este autor, el principal error de la interpretación de Ball consiste en pasar por alto el movimiento concreto de los precios de mercado que permite una inversión intensiva de capital, usando un modo de análisis "estático comparativo" en vez de uno "dinámico". En cambio, una vez que se considera este movimiento concreto resulta claro, según Evans, que el procedimiento ricardiano es el único correcto. El argumento básico es que, dado que el precio de mercado se eleva lentamente, antes de que se alcance el punto en que se pueda hacer una nueva inversión de capital se va formando una plusganancia que apropia efectivamente el terrateniente. En este contexto, si se asume el cálculo propuesto por Ball, la nueva inversión implica la desaparición de dicha plusganancia y, "no hay razón por la cual el terrateniente reduzca su renta a cero para permitir [...] la inversión" (Evans, 1992, p. 84, traducción propia). En cambio, si se adopta el cálculo ricardiano, todo lo que ocurre en esta situación es que la vieja inversión de capital continúa arrojando una plusganancia, mientras que la nueva no lo hace. Así, la inversión intensiva de capital en la producción agraria sólo puede explicarse bajo el supuesto del cálculo ricardiano. ${ }^{7}$

La crítica de Evans es correcta al nivel de las formas concretas del movimiento del capital. Sin embargo, antes de llegar a ese punto puede objetarse a Ball una deficiencia más general. El cálculo del precio de producción promedial o con base en el conjunto del capital adelantado que propone Ball implica que, de no existir la propiedad privada sobre la tierra, se debiera invertir cuotas de capital portadoras de una productividad del trabajo menor a la que determina el precio de mercado. Pero esto no significa otra cosa que gastar más trabajo social del que ya se gasta en producir las mercancías agrarias, lo cual no tiene ningún sentido desde el punto de vista del capital social global. Dicho de otro modo, no tiene sentido continuar aplicando cuotas de trabajo cada vez más improductivas sobre una misma tierra

${ }^{7}$ Esta crítica conducirá a Ball a matizar su interpretación, reconociendo que está basada en apenas uno de los varios ejemplos que pone Marx y que el enfoque del cálculo promedial tiene más relevancia para el caso de la renta urbana (Ball, 1992, p. 139). 
cuando se puede alcanzar el mismo producto con cuotas de trabajo más productivas, por ejemplo, las que se pueden aplicar sobre nuevas tierras. ${ }^{8}$

\section{IV.2 La interpretación moderna dominante}

Para la misma época en que Ball presenta su nueva lectura de la teoría marxiana de la RDII, se desarrolla una tercera interpretación que rápidamente se convertirá en la dominante dentro del marxismo y que, en este sentido, denominaremos interpretación moderna. Llamativamente, ésta se desarrollará de forma simultánea e independiente en la tradición hispanoamericana y en la anglosajona. ${ }^{9}$ Nos detendremos aquí en el análisis de sus autores originales, Guillermo Flichman (1977) en el primer caso, y Ben Fine (1979) en el segundo.

\section{La versión de Flichman}

La interpretación que ofrece Flichman de la RDII surge de una reformulación general de la presentación que hace Marx de la renta diferencial de tipo I (RDI). Más precisamente, de la pretensión de traducir dicha presentación "en una representa-

${ }^{8}$ En este sentido, a Ball parece caberle la misma crítica que Lenin le hacía a Bulgakov, esto es, que confunde "propiedad privada de la tierra" con "limitación de la tierra" (Lenin, 1981 [1901], p. 122 y ss.), ya que si no existiese la propiedad privada sobre la tierra, la asignación del trabajo social a la producción agraria permanecería sin cambio en lo que hace a la aplicación sucesiva de cuotas de trabajo, precisamente porque lo que seguiría existiendo es la limitación de las condiciones naturales, esto es, la fertilidad y la ubicación diferenciales de la tierra.

${ }^{9}$ Esta interpretación tiene al menos dos antecedentes que, sin embargo, no alcanzaron a difundirse como para siquiera desafiar la hegemonía de la interpretación clásica. El primero es el célebre Manual de Economía Política de la Academia de Ciencias Sociales de la URSS, en sus primeras dos ediciones (1956 [1954], p. 177; 1958 [1955], p. 187) que, aunque con matices y muy poca elaboración, presenta una lectura muy similar. Las ediciones subsiguientes del Manual, sin embargo, eliminan esta interpretación y vuelven a la interpretación clásica. El segundo antecedente es el de Michel Gutelman (1978 [1974]) que presenta una versión un poco más elaborada. Este autor comienza por distinguir tres tipos de renta diferencial, que recaen "sobre la fertilidad, sobre la ubicación y sobre la capitalización" (Gutelman, 1978 [1974], p. 94). Como veremos inmediatamente, es esta última la que va a coincidir con la interpretación moderna. En efecto, de acuerdo con Gutelman, una inversión adicional de capital podría incrementar más que proporcionalmente la producción, creando así una ganancia extraordinaria que podría embolsar el terrateniente: "Si el productor A (...) dobla su capital y, por esto, obtiene una producción mayor que el doble (...), tiene lugar entonces un plusbeneficio. Este plusbeneficio (...) podrá ser nuevamente captado por el propietario de la tierra en virtud de su derecho de propiedad" (Gutelman, 1978 [1974], pp. 104105). No existen evidencias, sin embargo, de que Flichman o Fine hayan leído el texto de Gutelman, cuya edición castellana data recién de 1978. 
ción geométrica", cuyo resultado es la inclusión del concepto clásico de la RDII en el concepto de la RDI (Flichman, 1977, p. 23).

Esta reformulación puede sintetizarse de la siguiente manera. Luego de plantear una serie de supuestos modeladores, como por ejemplo el de la inexistencia de cambio técnico, este autor hace una presentación donde el capital agrario aparece naturalmente dividido en múltiples partes, cada una de las cuales se pone en acción sucesivamente hasta el punto en que el rendimiento por unidad se iguala con aquel que determina el precio de mercado y que, como tal, es portador de una tasa de ganancial normal. De tal forma, señala:

[en] la tierra mejor se continuará invirtiendo mientras el rendimiento marginal de cada nueva inversión supere la cuota de ganancia media. La inversión se detendrá en el momento en que se iguale la ganancia proporcionada por la última unidad de inversión con la tasa media de ganancia. No hay razón alguna que indique que esto sucederá en el mismo nivel de inversión en los distintos tipos de tierra (Flichman, 1977, p. 25).

Sobre esta base concluye entonces que:

puede ampliarse la definición de renta diferencial I formulándola como la ganancia extraordinaria que surge por causa de la diferencia de fertilidad entre distintas tierras simultáneamente explotadas en forma capitalista, estando el valor comercial de los productos agrícolas fijado por el precio de producción de la tierra peor. No necesariamente el monto de la inversión por hectárea habrá de coincidir en las distintas tierras, más bien resulta lógico pensar que, en general, no coincidirá. (Flichman, 1977, p. 27). ${ }^{10}$

De este modo, lo que clásicamente se considera como la RDII queda subsumida bajo el concepto "ampliado" de RDI. Y, en efecto, el propio Flichman lo entiende en estos términos. "Cabe recordar", va a sostener posteriormente, "que mucho de lo que Marx expuso en los capítulos acerca de la renta diferencial II lo incluimos en nuestra formulación al tratar la renta diferencial I, y por ende queda dentro de una conceptualización acerca de la renta del suelo en el modo de producción capitalista" (Flichman, 1977, p. 54).

Sobre esta base, Flichman se ve forzado a reinterpretar el concepto de RDII. Para ello considerará las diferencias en la distribución del capital y el acceso al

${ }^{10}$ Una crítica a esta reformulación de la renta diferencial de tipo I puede verse en Klimovsky (1985, p. 101 y ss.). 
crédito entre los capitalistas agrarios debido al atraso relativo del capitalismo en la producción agraria. Así, de acuerdo con este autor, lo que se observa en:

casi todos los ejemplos [de Marx es] ... un grado de atraso relativo en el desarrollo del capitalismo en la agricultura, ya que no puede considerarse estable una situación en la que las inversiones adicionales están en condiciones inmediatas de obtención de beneficios extraordinarios, salvo que haya pocos capitales disponibles para ser invertidos en la rama agropecuaria. La mencionada situación permite que los capitalistas más avanzados obtengan ganancias extraordinarias [...] que al vencer los contratos de arrendamientos [...] pasan a convertirse en renta (Flichman, 1977, p. 23).

En consecuencia, concluye:

conviene considerar como renta diferencial II, solamente a la proveniente del atraso del desarrollo del capitalismo en la agricultura, que permite que el precio de producción individual para algunos arrendatarios capitalistas sea inferior al correspondiente a la peor tierra, no por ser más fértiles los terrenos en los que invierten su capital, sino por disponer de más recursos y mejor tecnología. (Flichman, 1977, p. 27).

En síntesis, para Flichman, la RDII está constituida específicamente por la ganancia extraordinaria que surge de alcanzar un precio de producción individual que se sitúa por debajo del precio de mercado en virtud de la puesta en acción de una mayor productividad del trabajo resultante, a su vez, de la aplicación de un capital de mayor tamaño al de los imperantes en la producción agraria. Más precisamente, la RDII está constituida por la apropiación por parte de la clase terrateniente de la ganancia extraordinaria que surge de la innovación tecnológica.

Antes de pasar al análisis de la versión de Fine de esta misma interpretación, conviene ya adelantar algunas de las implicancias o consecuencias problemáticas que conlleva la misma. En primer lugar, la existencia de la RDII depende aquí de la separación de la personificación de la propiedad de la tierra y del capital en dos individuos, ya que de otro modo el plusvalor extra se mantendría en la forma de plusganancia. En segundo lugar, la fuente de este tipo de renta no es, como en el caso de la RDI, una plusganancia que le corresponde genuinamente al propietario de la renta, sino una que le corresponde inicialmente al capitalista y luego es "confiscada" por el terrateniente. En este punto se pierde la raíz compartida con la renta diferencial de tipo I y, en consecuencia, el sentido de clasificarla como "de tipo II". En tercer lugar, como lo ha hecho notar recientemente un crítico de la interpretación de Flichman, esta interpretación implica que la tasa media de ganancia que determina los precios de las mercancías agrarias no se determina por las condiciones de producción del 
capital agrario "normal", esto es, del capital que se "requiere" "exactamente de la misma manera que en la industria ... a fin de poder elaborar las mercancías a su precio de producción" (Marx, 1997 [1865], p. 903), sino por un promedio entre la tasa de ganancia de este tipo de capital y las de los pequeños capitales agrarios, ya que de otro modo el primero no podría obtener la ganancia extraordinaria en cuestión (Iñigo Carrera, 2015, p. 291) ${ }^{11}$ Finalmente, y como se deduce de estas consideraciones, en esta interpretación la existencia de la RDII se muestra sujeta a la existencia de condiciones concretas muy particulares. Con todo, y en favor de la consistencia argumentativa de la interpretación de Flichman, debe notarse que él mismo es quien considera a la RDII como un concepto restrictivo a una situación histórica particular y que, en tal sentido, debe diferenciarse del concepto RDI, que "responde en forma más general a una definición de renta" (Flichman, 1977, p. 60).

\section{La versión de Fine}

A diferencia de Flichman, la interpretación de la RDII ofrecida por Ben Fine (1979) es más ambiciosa en cuanto al estatus teórico y filiación marxiana del concepto y, a consecuencia de ello, como veremos enseguida, también más problemática. Esta lectura tiene dos puntos de contacto básicos con la que realiza Ball. En primer lugar, Fine también parte de afirmar que "la intervención de la propiedad de la tierra modifica la formación del valor en la agricultura" (Fine, 1979, p. 251, traducción propia). En segundo lugar, y principalmente, coincide en poner en discusión la interpretación clásica de esta forma de renta por considerarla de índole ricardiana y, en consecuencia, contradictoria con la teoría marxiana del valor:

Lo que no puede aceptarse es que el análisis de Marx de la RDII es una extrapolación del [...] margen intensivo ricardiano [...] Si ésta fuera la teoría de Marx de la RDII, entonces requeriría el abandono de su teoría del valor, ya que el argumento [...] implica que el valor de cambio de las mercancías debería estar siempre determinado por el margen intensivo uniforme en lugar de por el valor de mercado (en general, el promedio de los valores individuales) (Fine, 1979, p. 251, traducción propia).

${ }^{11}$ La cuestión de la existencia de distintos tamaños de capital en la producción agraria y en particular de la persistencia de los pequeños capitales en ella ha estado en la base de la llamada "cuestión agraria" (Byres, 1991). Como se ha procurado argumentar en otro lugar, la presencia masiva de pequeños capitales en esta rama de la producción no afecta la determinación de los precios de las mercancías agrarias por la tasa general de ganancia y la renta de la tierra (Caligaris G., 2014). 
Sobre esta base, Fine desarrolla una interpretación que busca conciliar este "valor de mercado promedio" con las referencias textuales de Marx sobre las inversiones de capital sucesivas en la producción agraria. Su solución pasa por interpretar dicha sucesión de inversiones como distintos capitales individuales de distintos tamaños, los más grandes de los cuales pueden, en virtud de su mayor escala, producir una plusganancia que, a la postre, se transforma en renta de la tierra. En sus palabras,

Marx deja lo suficientemente claro en varios lugares que [... la RDII] depende de aplicaciones desiguales de capitales sobre las tierras [...]. El significado que da a estos capitales desiguales es su distinto tamaño como fuente de incremento de la productividad y ganancias extraordinarias [...] las ganancias extraordinarias que resultan de las inversiones de gran escala son sólo temporariamente prerrogativa del arrendatario capitalista, pero no por que vayan a ser erosionadas por la competencia con otros capitalistas. En última instancia, al igual que las ganancias extraordinarias que forman la RDI, son acumuladas por el terrateniente bajo la forma de la RDII (Fine, 1979, p. 251, traducción propia).

Para precisar y terminar de justificar esta explicación de la RDII, Fine sugiere considerar a dicha forma de renta en su "forma pura de inversiones desiguales de capital sobre tierras iguales", para lo cual supone que hay "una oferta ilimitada de tierra de igual calidad" (Fine, 1979, p. 252, traducción propia). Luego, bajo este supuesto, razona que:

de acuerdo a la teoría de Marx, la RDII se formaría a partir de inversiones de capital de un tamaño mayor al normal. Estos cultivos intensivos tendrían que dar por resultado economías de escala en el uso del capital, ya que de otra manera éste sería dividido y usado en una nueva tierra que no diera renta (Fine, 1979, p. 252, traducción propia).

Dando por finalizada la explicación sobre la RDII, Fine continúa la exposición presentando el vínculo de esta forma de renta con su antecesora, vale decir, sin considerar ya a la RDII en su forma pura. Entonces, y como consecuencia de haber levantado el supuesto de la oferta ilimitada de tierras iguales, enfrenta el problema de cómo determinar el tamaño normal del capital, pues, según su interpretación, "algunos capitales podrían ser normales para determinados tipos de tierra, y otros para otras" (Fine, 1979, p. 254, traducción propia); problema que, inmediatamente, se le traslada a la determinación de la peor tierra, pues, también según su interpretación, "algunas tierras podrían ser peores para determinados niveles de 
inversión, pero no para otros" (Fine, 1979, p. 254, traducción propia). La solución que ofrece a este problema pasa por considerar "la determinación simultánea de la tierra peor y el capital normal" (Fine, 1979, p. 255, traducción propia) donde, partiendo de que "la propia estructura de rentas interviene en la formación de la estructura de acumulación del capital" (Fine, 1979, p. 256, traducción propia) acaba por concluir que "la estructura de acumulación del capital será influenciada por la estructura de rentas, en la misma medida que la formará" (Fine, 1979, p. 257, traducción propia), dejando a la cuestión concreta del tamaño del capital normal y la peor tierra, en la abstracta -y por cierto muy neoclásica- determinación simultánea. ${ }^{12}$

A modo de corolario, Fine acaba justificando esta interpretación de la renta RDII por su potencialidad para explicar el polémico supuesto de la baja composición orgánica del capital agrario que constituye la base de la renta absoluta (Fine, 1979, p. 262). En efecto, según esta interpretación, al quedarse con la plusganancia que surge del aumento de la productividad del trabajo, la propiedad de la tierra desincentiva la inversión de capital y con ello acaba por constituirse en una barrera a la tecnificación del capital.

Como se puede apreciar, la lectura de Fine de la RDII no se diferencia esencialmente de la de Flichman. En este sentido, le corresponden las mismas críticas que ya hemos hecho a la interpretación de este último autor. Sin embargo, como se

${ }^{12}$ En contraposición, años más tarde, en su célebre Marx's Capital publicado en los últimos años junto a Saad-Filho, reconocerá explícitamente la imposibilidad teórica de resolver el problema:

El problema de la determinación conjunta del capital normal y la tierra peor (o, más exactamente, la tierra normal, ya que la tierra en uso físicamente peor podría no ser la que determine el valor) no puede ser resulto abstractamente; por lo tanto, la RDI y la RDII no pueden ser determinadas de forma puramente teórica [...] depende de condiciones históricamente contingentes, de cómo la agricultura se ha desarrollado en el pasado y cómo se relaciona con la acumulación del capital en términos del acceso a la tierra de los capitalistas [...]. Más aún, los cambios en los cultivos y las tecnologías de producción modifican la demanda de tierra, y las definiciones de la tierra mejor o peor. En síntesis, la teoría de la RD no conduce específicamente a un determinado análisis de la renta, pero revela los procesos por los cuales puede ser examinada históricamente. (Fine y Saad-Filho, 2010 [2004], p. 160, traducción propia).

En la presentación de Harvey, este punto también es bastante oscuro:

Las complejas interacciones de la RD-1 [...] y de la RD-2 [...] hacen imposible distinguir qué es lo que debe obtener cada uno de ellos [capitalistas y terratenientes]; las relaciones reales se vuelven borrosas. [...]. Lo que al principio parecía un instrumento pulcro para racionalizar la coordinación de la inversión en la tierra, se convierte en una fuente de contradicción, confusión e irracionalidad. (Harvey, 1990 [1982], pp. 362-365). 
puede reconstruir a partir de algunas críticas específicas realizadas al enfoque de Fine, se presentan en esta versión de la interpretación moderna problemas aún más numerosos y agudos que en la de Flichman. Considerémoslos brevemente. En primer lugar, centrar la explicación en el supuesto de que "toda la tierra es igual y en cantidad ilimitada" carece de sentido toda vez que precisamente lo que está en juego en la producción agraria es la limitación y la desigualdad en las calidades de las tierras; es, en suma, "negar la condición fundamental de la existencia de la relación de renta" (Ball, 1980, p. 310, traducción propia). En segundo lugar, el hecho de que las plusganancias existan sólo de forma temporaria, niega el concepto mismo de renta de la tierra (Ball, 1980, p. 310). En tercer lugar, el desincentivo a la inversión que resultaría de este proceso es relativo, ya que no sólo sería posible que las ganancias extraordinarias fueran mayores que la renta pagada, sino que tal situación desaparecería en el caso de que el capitalista y el terrateniente fueran la misma persona (Ball, 1980, p. 310). En cuarto lugar, lejos de salvar al "concepto de valor de mercado" de las contradicciones de la economía neoclásica, esta interpretación lo deja en un completo vacío de determinación, atrapado entre la determinación por la peor tierra y la determinación por el capital normal, como lo pone Ball:

¿Cómo se determina el valor de cambio? Parecerían existir dos procesos distintos: la determinación del peor suelo por la RDI, y la determinación del capital normal por la RDII. Ambos no pueden ser el mismo, ya que distintas magnitudes de capital serán invertidas en distintas tierras. Fine sugiere, por ejemplo, que cada tierra tendrá su propio capital normal. ¿Cuál de ellos va entonces a determinar el precio de mercado? (Ball, 1980, p. 311, traducción propia). ${ }^{13}$

Por su parte, en la reciente crítica de Iñigo Carrera, encontramos otra objeción de peso a la interpretación de Fine. Según esta crítica, luego de concluir que la RDII surge de la mayor escala con que se aplica el capital agrario, lo que Fine debería explicar es "cómo es posible que coexistan dos cánones de arriendo diferenciados para exactamente la misma tierra" (Iñigo Carrera, 2015, p. 301). Más concretamente,

\footnotetext{
${ }^{13}$ Esta serie de objeciones no han sido respondidas aún por Fine. En una débil defensa de su argumento, este autor se limitó a señalar que su interpretación es más consistente porque, a diferencia de la de Ball, supone el dominio de la producción capitalista sobre la agricultura; por otra parte, establece como eje central de la discusión la cuestión de si la propiedad de la tierra invalida o no la estructura de relaciones económicas asociadas con el capital o simplemente modifica estos efectos, y dictamina que el problema no puede resolverse de forma teórica, sino que se trata de una cuestión histórica (Fine, 1980).
} 
debería explicar bajo qué formas concretas podría existir un canon de arriendo diferenciado que impusiera un mayor pago a los capitales más concentrados y, como tales, de mayor poder económico frente a los terratenientes, mientras los capitales menores, económicamente más débiles, pagarían alegremente un canon menor (Iñigo Carrera, 2015, p. 301).

Finalmente, como veremos más adelante, la evidencia textual que presenta Fine para sostener la filiación marxiana de su interpretación no pasa de unas pocas, escuetas y descontextualizadas citas. En el mejor de los casos, su interpretación, como también señala Ball, "se acerca a uno de los catorce casos considerados por Marx" (Ball, 1980, p. 310, traducción propia). Y a la inversa, las numerosas referencias textuales que contradicen su interpretación no son discutidas.

\section{La difusión masiva y la naturalización de la interpretación moderna}

A pesar de las debilidades argumentativas de la interpretación moderna, su difusión fue rápida y masiva.

En lo que respecta a la literatura anglosajona, la versión de Fine tuvo inmediatamente una buena recepción (por ejemplo, Neocosmos (1982) y Harvey (1982)), que posteriormente se masifica, en especial a través de las sucesivas reediciones de los populares trabajos de David Harvey (1990 [1982]) y del propio Fine y Saad-Filho (2010 [2004]). ${ }^{14}$ En la actualidad, se la acepta casi sin excepciones como la única interpretación válida de este tipo de renta (por ejemplo, Vlachou (2002); Campbell (2002); Ramírez (2009); Park (2010; 2014); Munro (2012) y Campling y Havice (2014)), y cada vez es más difícil encontrar trabajos que remitan a sus autores originales o que la presenten como una interpretación en disputa (por ejemplo, Halia (1990) y Jäger (2003)). Más aún, la interpretación moderna ha permeado hasta en los autores críticos de la teoría de la renta de Marx (por ejemplo, Bryan (1990)).

De manera similar, en la tradición latinoamericana, la versión de Flichman ha gozado de amplia aceptación. Inicialmente, se la puede ver acogida con cierto entusiasmo (Margulis, 1979; Kamppeter, 1983), manifiesto rechazo (Klimovsky, 1985) e indiferencia (Mendoza, 1985), según los casos. Pero pasado unos

\footnotetext{
${ }^{14}$ En esta difusión también es destacable el papel de los dos de los diccionarios marxistas más importantes de la actualidad: A Dictionary of Marxist Thought, editado por Tom Bottomore (1983 [1991]), y el Dictionnaire critique du marxisme, editado por Gérard Bensussan y Georges Labica (1999 [1982]). En ambos se presenta acríticamente a la interpretación moderna como si fuese la única interpretación existente. En el primer caso, el encargado es el propio Ben Fine (1991 [1983], p. 302), en el segundo, Marcel Drach (1982 [1999], p. 620).
} 
años, se la comienza a asumir como la única interpretación existente, en algunos casos remitiendo al trabajo de Flichman (Kabat, 1999; Rodríguez, 2004), y en otros con completa omisión de éste (Moreira, 1995; Farina, 2006; Balsa, 2006; Sartelli, 2008; Anino y Mercatante, 2009; Astarita, 2010; de Melo Faria, 2012; Oyhantçabal y Narbondo, 2013). Finalmente, con el borrado ya absoluto de toda controversia y fuentes interpretativas, e instalada como un lugar común en las consideraciones teóricas sobre la renta de la tierra, se llega incluso al punto de invertirse completamente la historia del desarrollo interpretativo, presentando a la interpretación clásica como si fuera moderna y a ésta como si fuera clásica (Astarita, 2009; Fernández, 2010). En autores que hacen este tipo de operaciones la confusión en la que recaen es realmente notable, ya que son precisamente aquellos que consideran al concepto de RDII de "importancia fundamental" para comprender la evolución actual de la producción agraria. Casos excepcionales a esta tendencia general son los trabajos de Plascencia (1995), Arceo (2003) e Iñigo Carrera $(2007 ; 2015)$.

\section{UN BALANCE CRÍTICO DE LAS INTERPRETACIONES SOBRE LA RDII A LA LUZ DE LA EVIDENCIA TEXTUAL PRESENTE EN LA CRÍTICA MARXIANA DE LA ECONOMÍA POLÍTICA}

A lo largo de nuestra reseña crítica de la historia de la recepción de la explicación marxiana de la RDII hemos señalado las principales insuficiencias y debilidades de las tres interpretaciones identificadas. En esta sección reconsideraremos la fortaleza de cada una de estas interpretaciones a la luz de la evidencia textual disponible en la obra de Marx.

Hemos visto que un aspecto sensible en todas las lecturas de la explicación marxiana de la RDII es la vinculación que tiene con la explicación ofrecida por Ricardo. Como se recordará, mientras que para la interpretación clásica ambas explicaciones son esencialmente iguales y por lo tanto no es necesario realizar mayores elaboraciones al respecto, para las dos interpretaciones modernas la diferencia entre las mismas es radical y, más importante aún, en los casos de Ball y Fine se presenta a dicha diferencia como el eje en torno al cual gira la explicación marxiana de la RDII. En este punto, un examen crítico de los textos de Marx al respecto cuestiona seriamente la lectura de las interpretaciones modernas.

Ante todo, en sus tempranas y pormenorizadas lecturas de la teoría ricardiana de la renta de la tierra, Marx señala que Ricardo reconoce la existencia de una renta diferencial producto de la inversión de una "porción adicional de capital, con un producto menor, a la misma tierra" (Marx, 1998 [1851], p. 35), estando el precio 
del producto determinado por aquél que involucra la "mayor cantidad de trabajo" (Marx, 1987 [1847], p. 107); esto es, interpreta que Ricardo formula una explicación de la RDII tal como ulteriormente la presentará la que aquí llamamos "interpretación clásica". Sobre esta base, lo llamativo es que de las numerosas críticas que Marx hace de la teoría ricardiana de la renta ninguna apunta contra la explicación que ofrece Ricardo de este tipo de renta diferencial (Marx, 1987 [1847], p. 109; 1987 [1862-1863], pp. 213-225, 277-310, 355-363; 1983 [1845-1895], pp. 38, 120-123, 130, 249-259; 1997 [1865], pp. 847, 861, 872, 874, 981; véase una síntesis en Gehrke, 2012). Sólo en una de ellas, Marx critica a Ricardo que "redu[ce] toda la formación de la renta diferencial II" a un "único caso" en que sube el "precio de producción" (Marx, 1997 [1865], p. 874); esto es, lo critica, tal como hemos visto que se lo hace desde la interpretación clásica, por la insuficiencia con la que trata las relaciones cuantitativas en la que se expresa este tipo de renta. Por otra parte, es notable que las pocas veces en que Marx señala las implicancias del enfoque de Ricardo sobre la determinación de los precios en el análisis de la renta de la tierra lo hace exclusivamente en relación con la "renta absoluta" y no a la RDII. Más aún, cuando lo hace, deja explícitamente de lado toda consideración sobre la renta diferencial (Marx, 1987 [1862-1863], pp. 216 y ss.). En este punto, resulta cuanto menos llamativo que las interpretaciones modernas no se hayan preocupado por discutir esta evidencia de la crítica de Marx a Ricardo ni por presentar otros pasajes de esta crítica que validen su lectura.

En particular, en la interpretación moderna que se ha convertido actualmente en dominante, la discusión de los pasajes de Marx que cuestionan su lectura, al menos considerados restringidamente, es prácticamente inexistente (véase, especialmente, Marx, 1997 [1865], pp. 865, 926). En cambio, esta interpretación presenta dos citas de Marx como evidencia sumaria de su filiación a la explicación marxiana de la RDII. Considerémoslas brevemente. En la primera de ellas, Marx señala que:

la diferente magnitud de la renta de la tierra, partiendo de la misma inversión de capital, sólo puede explicarse por la diferente fertilidad de las tierras. La diferente magnitud de la renta de la tierra, cuando la fertilidad es igual, no tiene más explicación que por la diferente magnitud de la inversión de capital (Marx, 1987 [1862-1863], p. 32, traducción modificada; citado en Fine, 1979, p. 251).

Ante todo, hay que notar que Marx está escribiendo en un contexto en donde no está presentando ni discutiendo la renta diferencial. Por ese motivo, se trata de una expresión muy sintética, y en tal sentido puramente analítica, de las dos formas de renta diferencial. Pero fundamentalmente, hay que notar que, en esta síntesis, la interpretación clásica no se ve contradicha: inversiones sucesivas de capital, 
portadoras de una productividad del trabajo decreciente, se manifiestan como magnitudes diferentes del capital adelantado en cada proceso de producción, y es esa magnitud la que explica, cæterīs pāribus las diferencias de fertilidad entre las tierras, la magnitud de la renta.

La segunda cita a la que acuden los autores de la interpretación moderna, y que luego es repetida insistentemente por quienes subscriben a la misma, es la siguiente:

en la renta diferencial en la forma II se suman, a diferencia de la fertilidad, las diferencias en la distribución del capital (y de capacidad de crédito) entre los arrendatarios. (Marx, 1997 [1865], p. 869; citado en Flichman, 1977, p. 22 y Fine, 1979, p. 252).

Aquí, una vez más, conviene empezar por precisar el contexto en que aparece este texto. Marx ya ha definido a la RDII (Marx, 1997 [1865], pág. 865) y, como acostumbra hacer en sus borradores, está realizando algunos comentarios adicionales. En el manuscrito original, de hecho, entre esta primera definición y la cita en cuestión, Marx escribe unos "agregados" que en la edición de Engels constituyen las páginas finales del capítulo anterior (Marx y Engels, 1992, pp. 780-786; 857863 de la edición castellana citada). Y es recién después de ellos que Marx comenta que "al considerar la renta diferencial II aún es necesario destacar los puntos siguientes" (Marx, 1997 [1865], p. 867): el "Primero" referido a que la RDII es, pace Fine, inseparable de la RDI porque ésta constituye "su base y punto de partida" (Marx, 1997 [1865], p. 867); y el "Segundo" es precisamente el comentario que recogen Flichman y Fine. Se trata, por consiguiente, de una nota marginal, vale decir, un comentario que no hace al desarrollo sistemático de la crítica marxiana. El contenido de esta referencia se aclara a poco que el lector avanza más allá del texto citado. Marx simplemente quiere llamar la atención respecto a que, cuando se va a analizar, en un país dado, la cuestión de las inversiones sucesivas de capital, es necesario recordar que "[e]1 modo capitalista de producción sólo se apodera en forma lenta y despareja de la agricultura" (Marx, 1997 [1865], p. 869), y que, por tanto, no se puede suponer que todos los capitales son iguales, luego de lo cual resulta relevante tener en cuenta estas diferencias para no confundirlas con las que derivan de la presencia de inversiones sucesivas de capital que dan lugar a la RDII.

En su detallada lectura de la sección sexta de El Capital, Diehl (1899) ha sostenido esencialmente esta misma interpretación del pasaje de Marx en cuestión; aunque, en su caso, con el objetivo criticar a Marx por confundir niveles de análisis, "perdiéndose en gran parte la claridad teórica y nitidez de la teoría ricardiana" (Diehl, 1899, p. 465, traducción propia). Recientemente, Iñigo Carrera también ha sugerido una lectura similar del texto marxiano en cuestión. En sus palabras: 
La renta diferencial II no brota de una diferencia en la magnitud absoluta del capital aplicado sobre la tierra sino de las diferencias en la productividad que resultan de la aplicación sucesiva de las porciones que integran técnicamente dicho capital. Pero es obvio que para poder alcanzar la intensidad de aplicación plena de la cual surge la plenitud de esta renta diferencial, el capitalista agrario debe disponer de la masa íntegra de capital correspondiente. Marx señala [en el pasaje discutido] esta circunstancia, ajena en sí misma a la determinación de la renta diferencial II, pero que media en la apropiación plena de la misma (Iñigo Carrera, 2015, pp. 287-288).

\section{Conclusiones}

En nuestra reconstrucción crítica de la historia de las interpretaciones marxistas de la RDII hemos visto que en las últimas décadas la interpretación moderna desarrollada originalmente por Flichman y Fine ha conquistado un grado tal de hegemonía que se la ha llegado a naturalizar como la única existente. Siendo la RDII un momento clave de la explicación de la forma específica que adopta la inversión del capital agrario, y como tal inescapable para una explicación de la actualidad de la producción agraria, en este trabajo nos hemos propuesto recuperar la historia de las interpretaciones marxianas en torno a esta forma de renta y los debates que éstas originaron.

En efecto, como se puede apreciar luego de nuestra reconstrucción crítica, cada interpretación implica, cuando menos, un análisis distinto del curso de la inversión del capital agrario. Por ejemplo, según la interpretación moderna actualmente dominante, una situación de baja intensidad en la inversión de capital en la producción primaria se podría explicar por el poder terrateniente sobre las potenciales plusganancias provocadas por una inversión intensiva de capital. A la inversa, una situación de alta intensidad en la aplicación de capital se podría explicar por la posibilidad del capitalista de retener dicha plusganancia. En cambio, según la interpretación clásica, una situación de baja intensidad en la inversión de capital se explica esencialmente por una caída en el precio de mercado, que impide la aplicación de la última inversión de capital a la tasa normal de ganancia. Y a la inversa, el caso de una situación de alta intensidad en la inversión de capital, se explica por una suba del precio de mercado.

Sinteticemos los resultados de nuestra reconstrucción crítica de la historia de las interpretaciones marxistas de la RDII. En cuanto a las interpretaciones modernas, ante todo hemos mostrado que la evidencia textual de la obra de Marx en la que se apoyan es sumamente débil. Más importante aún, hemos identificado problemas sustantivos en sus argumentaciones. En el caso de Ball, en cuanto el cómputo 
promedial que propone, aun dejando a un lado su irrelevancia práctica, comporta el problema de suponer la puesta en acción de un trabajo aún más improductivo que el que se tiene que poner para satisfacer la demanda social. En el caso de Flichman, en cuanto su argumento apenas si puede lograr consistencia, de un parte, eliminando la problemática de la inversión sucesiva de capital presentándola bajo un modelo restrictivo dentro de la RDI y, de otra parte, resignando a la RDII a situaciones concretas extremadamente particulares. En el caso de Fine, finalmente, en cuanto su argumento sólo cobra sentido eliminando precisamente lo que hace específico al movimiento del capital en la producción agraria, esto es, la limitación de las condiciones naturales de producción susceptibles de control por el capital. A su vez, como hemos visto, en los tres autores la generación de la renta de la tierra en cuestión se explica siempre por la existencia de la clase terrateniente separada inmediatamente de la clase capitalista, cuando por definición esta separación es sólo condición para la transmutación formal de una plusganancia ya generada en renta de la tierra propiamente dicha.

En contraposición, hemos visto que la interpretación clásica, si bien puede ser objetada por hallarse insuficientemente desarrollada, no encuentra objeciones sustantivas ni se contrapone a los fundamentos de la crítica de la economía política. En este sentido, pensamos que para avanzar en la compresión de los fenómenos de inversión intensiva del capital en la producción primaria desde la perspectiva de la crítica marxiana es necesario reponer y desarrollar esta lectura de la RDII. En particular, si consideramos la línea de investigación abierta por Marx, este desarrollo debe pasar por la sistematización de las relaciones cuantitativas entre las inversiones de capital, la productividad del trabajo, el tamaño de la necesidad social y la renta de la tierra a las que da lugar la existencia de este tipo de renta diferencial.

\section{REFERENCIAS BIBLIOGRÁFICAS}

Academia de "Ciencias de la URSS (1956 [1954]), Manual de Economía Política, México, Grijalbo. (1958 [1955]), Manual de economía política, México, Grijalbo.

Amin, S. y Vergopoulos, K. (1977 [1974]), La cuestión campesina y el capitalismo, México, Nuestro Tiempo.

Anino, P. y Mercatante, E. (julio de 2009), Renta diferencial y produccion agraria en Argentina. Una respuesta a Rolando Astarita, recuperado en julio de 2009, de IPS - Instituto del pensamiento socialista: http://www.ips.org.ar/ 
wp-content/uploads/2011/04/Anino-y-Mercatante-Renta_diferencial_y_ produccion_agraria_en_Argentina.pdf

Arceo, E. (2003), Argentina en la periferia próspera. Renta internacional, dominación oligárquica y modo de acumulación, Bernal, Universidad Nacional de Quilmes.

Arthur, C. (1997), "Against the Logical-Historical Method: Dialectical Derivation versus Linear Logic", en F. Moseley y M. Campbell, New Investigations of Marx's Method, New Jersey, Humanities Press, 9-37.

Arthur, C. J. (2006), "The Inner Totatity”, Historical Materialism, 14 (03), 85-111. Astarita, R. (2010), Economía política de la dependencia y el subdesarrollo. Tipo de cambio y renta agraria en la Argentina, Bernal, Universidad Nacional de Quilmes.

(Junio de 2009b), Renta agraria, ganancia del capital y tipo de cambio, recuperado en julio de 2009, de IPS - Instituto del pensamiento socialista: http://www.ips.org.ar/wp-content/uploads/2011/04/Astarita-Rolando-Renta-ganancia-y-tipo-de-cambio-11.pdf

(septiembre de 2009a), Respuesta al profesor Juan Iñigo Carrera, recuperado en septiembre de 2009, de IPS - Insittuto del pensamiento socialista: http://www.ips.org.ar/wp-content/uploads/2011/04/Rolando-Astarita-Respuesta_a_Inigo_Carrera.pdf

Ball, M. (1992), "Land at the margin? A comment on Evans, 'On differential rent and landed property", International Journal of Urban and Regional Research, vol. 16, $\mathrm{N}^{\mathrm{o}} 1,138-141$.

(1977), "Differential rent and the role of landed property", International Journal of Urban and Regional Research, vol. 1, No 1-4, 380-403.

(1980), "On Marx's theory of agricultural rent: A reply to Ben Fine", Economy and Society, 9 (3), 304-326.

Backhaus, H. G. (1978 [1969]), "La dialéctica de la forma de valor", Dialéctica (04), 9-34.

Balsa, J. (2006), El desvanecimiento del mundo chacarero. Transformaciones sociales en la agricultura bonaerense, Bernal, Universidad Nacional de Quilmes.

Barnes, T. (1984), "Theories of Agricultural Rent within the Surplus Approach". International Regional Science Review, vol. 9, No 2, 125-140.

Bartra, A. (1979 [1976]), "La renta capitalista de la tierra", Cuadernos Agrarios 7 (8), 41-112. 
Bensussan, G. y Labica, G. (1999 [1982]), Dictionnaire critique du marxisme, Paris, Quadrige / Puf.

Bernstein, H. (2012), Dinámicas de clase y transformación agraria, México, UAZICAS-PORRÚA.

Borchardt, J. (1981 [1919]), El Capital de Marx. Versión abreviada, México, Sanchez Mato.

Bortkiewicz, L. (1910-11), "La teoría de la renta de la tierra de Rodbertus y la doctrina de Marx acerca de la renta de la tierra absoluta", Cuadernos Agrarios, 4, 169-235, 1979.

Bottomore, T. (1983 [1991]), A Dictionary of Marxist Thought, Oxford, Blackwell Publishers.

Bryan, D. (1990), “'Natural', and 'Improved' Land in Marx's Theory of Rent”, Land Economics, 66 (2), 176-181.

Byres, T. J. (1991), Agraria Question, en T. Bottomore, A Dictionary of Marxist Thought, Oxford, Blackwell Publishers, 9-11.

Caligaris, G. (2014), Acumulación de capital y estructura social en la producción agraria pampeana (1996-2013), Buenos Aires, tesis doctoral, Facultad de Ciencias Sociales de la Universidad de Buenos Aires. (2014), Estructura social y acumulación de capital en la producción agraria pampeana (1996-2013), tesis de doctorado, Facultad de Ciencias Sociales de la Universidad de Buenos Aires, Buenos Aires.

Caligaris, G. y Starosta, G. (2017), "La determinación actual e histórica en la génesis del dinero. Una aproximación metodológica a la controversia sobre el carácter mercantil de la forma dineraria”, en R. Escorcia Romo, y M. Robles Baez, Dinero y Capital. Hacia una Reconstrucción de la Teoría del Dinero de Marx, México, Universidad Autónoma Metropolitana, 123-157. (2016), "La determinación actual e histórica en la génesis del dinero: Una aproximación metodológica a la controversia sobre el carácter mercantil de la forma dineraria", en R. Escorcia Romo y M. L. Robles Baez (edits.), Dinero y Capital. Hacia una Reconstrucción de la Teoría del Dinero de Marx, México, Universidad Autónoma Metropolitana, 92-122.

Campling, L. y Havice, E. (2014), "The problem of property in industrial fisheries", The Journal of Peasant Studies, 41 (5), 707-727.

Campbell, M. (2002), "Rent and Landed Property", en M. Campbell y G. Reuten, The Culmination of Capital. Essays on Volume III of Marx's Capital, Houndmills, Basingstoke, Palgrave, 228-241. 
De Melo Faria, A. M. (2012), "O ponto perdido na trama do desenvolvimento da cotonicultura em Mato Grosso", Novos Cadernos NAEA, 15 (1), 161-178.

De Vroey, M. (1982), "On the Obsolescence of the Marxian Theory of Value: A Critical Review", Capital \& Class, 6 (2), 24-59.

Diehl, K. (1899), "Die Grundrententheorie im okonomischen System von Karl Marx", Jahrbiicher fur Nationalokonomie und Statistik, Bd. XVII, Dritte Folge, 433-80.

Drach, M. (1982 [1999]), "Investissement", en G. Bensussan y G. Labica, Dictionnaire critique du marxisme, Paris, Quadrige/Puf, 619-621.

Elson, D. (1979), Value: The Representation of Labour in Capitalism: Essays. London, CSE Book.

Emmanuel, A. (1972 [1969]), El intercambio desigual. Ensayo sobre los antagonismos en las relaciones económicas internacionales, México, Siglo XXI.

Engels, F. (1997 [1859]), "La 'Contribución a la crítica de la economía política' de Karl Marx”, en K. Marx, Contribución a la crítica de la economía politica, México, Siglo XXI, 333-343.

Evans, A. W. (1992), "On Differential Rent and Landed Property”, International Journal of Urban and Regional Research, 81-96.

Flichman, G. (1977), La renta del suelo y el desarrollo agrario argentino, Buenos Aires, Siglo XXI.

Flichman, G., Hagedoorn, L. y Stroom, J. (1981), Renta del suelo y economía internacional, Amsterdam, CEDLA.

Farina, J. (2006), "El concepto de renta: un análisis de su versión clásica y marxista", VIII Reunión de Economía Mundial, Alicante, Sociedad de Economía Mundial, 1-30.

Fernandez, D. (2010), "Reflexiones sobre el crecimiento agrícola pampeano a la luz del concepto de la renta diferencial de segundo tipo", Documentos del CIEA, $\mathrm{N}^{\circ} 6,5-29$.

Fine, B. (1991 [1983]), "Landed property and rent", en T. Bottomore, A Dictionary of Marxist Thought, Oxford, Blackwell Publishers, 302-303.

(1979), “On Marx's theory of agricultural rent”, Economy and Society, 8 (3), 241-278.

(1980), "On Marx's theory of agricultural rent: A rejoinder", Economy and Society, 9 (3), 327-331.

Fine, B. y Saad-Filho, A. (2010 [2004]), Marx's Capital, London, Pluto Press. 
Fineschi, R. (2013), “The Four Levels of Abstraction of Marx's Concept of 'Capital'. Or, Can We Consider the Grundrisse the Most Advanced Version of Marx's Concept of Capital?", en R. Bellofiore, P. D. Thomas y G. Starosta, In Marx's Laboratory. Critical Interpretations of the Grundrisse, Leiden, Brill, 71-98.

Gehrke, C. (2012), "Marx's critique of Ricardo's theory of rent: a re-assessment", en N. Salvadori, C. Gehrke, I. Steedman y R. Sturn (edits.), Classical political economy and modern theory: Essays in honour of Heinz Kurz, London, Routledge, 51-85.

Grossmann, H. (1979 [1929]), "Modificación del plan original de la estructura de 'El Capital"', en H. Grossmann, Ensayos sobre la teoría de las crisis. Dialéctica y metodología en "El capital”, México, Pasado y Presente, 41-70.

Guidi, M. E. (1998), "Land Rent and the Logic of Capital", en R. Bellofiore (ed.), Marxian Economics: A Reappraisal. Essays on Volume HI of Capital, Houndmills, Basingstoke, Macmillan Press, 71-93.

Gutelman, M. (1978 [1974]), Estructuras y reformas agrarias, Barcelona, Fontamara.

Halia, A. (1990), "The theory of land rent at the crossroads", Environment and Planning D: Society and Space, vol. 8, 275-296.

Harvey, D. (1982), "Land Rent and the Transition to the Capitalist Mode of Production", Antipode, vol. 14, No 3, 17-25. (1990 [1982]), Los límites del capitalismo y la teoría marxista, México, Fondo de Cultura Económica.

Howard, M. C. y King, J. E. (1988), The political economy of Marx, New York, NYU Press.

Iñigo Carrera, J. (2015), La especificidad nacional de la acumulación de capital en la Argentina: desde sus manifestaciones originarias hasta la evidencia de su contenido en las primeras décadas del siglo XX, Buenos Aires, tesis doctoral, Facultad de Filosofía y Letras de la Universidad de Buenos Aires.

(2007), La formación económica de la sociedad argentina. Volumen I, Renta agraria, ganancia industrial y deuda externa. 1882-2004, Buenos Aires, Imago Mundi.

(julio de 2009), Renta agraria, ganancia del capital y tipo de cambio: respuesta a Rolando Astarita, recuperado en julio de 2009, de IPS - Instituto del pensamiento socialista: http:/www.ips.org.ar/wp-con tent/ 
uploads/2011/04/Juan_Inigo_Carrera_Respuesta_a_Astarita_so bre_ renta.pdf

Jäger, J. (2003), "Urban Land Rent Theory: A Regulationist perspective", International Journal of Urban and Regional Research, 27 (2), 233-249.

Kabat, M. (1999), "La renta diferencial y el desarrollo del sector chacarero". Razón y Revolución, $\mathrm{N}^{\mathrm{o}}$ 5, reedición electrónica, 1-11.

Kamppeter, W. (1983), "La renta del suelo", en H. Lefevre, J. M. Caballero, O. González, y W. Kamppeter, La renta de la tierra. 5 Ensayos, México, Tlaiualli, 113-128.

Kautsky, K. (2002 [1899]), La cuestión agraria, México, Siglo XXI.

Kicillof, A. y Starosta, G. (2007a), "Value form and class struggle: A critique of the autonomist theory of value", Capital \& Class, 31 (2), 13-40. (2007b), "On Materiality and Social Form: A Political Critique of Rubin's Value-Form Theory", Historical Materialism, 15 (3), 9-43.

Klimovsky, E. (1985), Renta y ganancia en al economía política clásica, México, Universidad Autónoma Metropolitana, Azcapotzalco.

Kloppenburg, J. R. (2004), First the Seed: The Political Economy of Plant Biotechnology, 1492-2000, Wisconsin, The University of Wisconsin Press.

Lapidus, I. y Ostrovitianov, K. (1929), An outline of political economy: political economy and soviet economics, London, Martin Lawrence.

Lenin, V. I. (1981 [1901]), "El problema agrario y los “críticos de Marx", en V. I. Lenin, Obras completas, vol. 5, Moscú, Progreso, 99-283. (1983 [1908]), "El programa agrario de la socialdemocracia en la primera revolución rusa de 1905-1907”, en V. I. Lenin, Obras completas, vol. 16, Moscú, Progreso, 221-440.

Mandel, E. (1972 [1962]), Tratado de economía marxista, t. I, México, Era.

Margulis, M. (1979), Contradicciones en la estructura agraria y transferencias de valor, México, El Colegio de México.

Marx, K. (1983 [1845-1895]), Cartas sobre “El Capital”, La Habana, Editora Política.

(1997 [1859]), Contribución a la crítica de la economía política, México, Siglo XXI.

(1997 [1865]), El Capital. Crítica de la economía política, t. III, vol. 8, México, Siglo XXI.

(1997), Elementos fundamentales para la crítica de la economía política (Grundrisse) 1857-1858. vol. 1, México, Siglo XXI. 
(1987 [1847]), Miseria de la filosofía. Respuesta a la Filosofía de la Miseria de P. J. Proudhon, México, Siglo XXI.

(1998 [1851]), "Notas y extractos sobre el sistema de Ricardo. Marzo-abril de 1851", en Elementos fundamentales para la crítica de la economía política (Grundrisse) 1857-1858, vol. 3, México, Siglo XXI, 23-88. (1987 [1862-1863]), Teorías sobre la plusvalía II, t. IV de El Capital. México, Fondo de Cultura Económica.

Marx, K. y Engels, F. (1992), Gesamtausgabe (MEGA), Abt. 2. Bd. 4 Text: Karl Marx ökonomische Manuskripte 1863-1867, Berlin, Dietz.

Mendoza, C. (1985), Teoría de la renta capitalista de la tierra, Buenos Aires, Ateneo.

Mercatante, E. (diciembre de 2010), La naturaleza de la renta agraria en Argentina. Los efectos de su apropiación parcial vía retenciones (2002-2008), recuperado en mayo de 2011, de IPS - Instituto del pensamiento socialista: http://www.ips.org.ar/wp-content/uploads/2011/04/Mercatante_Esteban_La_naturaleza_de_la_renta_agraria_en_la_Argentina._Los_efectos_de_su_apropiaci\%C3\%B3n_parcial_mediante_retenciones_2002-2008.pdf

(Julio de 2009), La renta diferencial y su impacto en el capitalismo argentino, recuperado en julio de 2009, de http://www.ips.org.ar/?p=1062

Moreira, R. J. (1995), "Renda da natureza e territorialização do capital: reinterpretando a renda da terra", Estudos Sociedade e Agricultura, 4, 89-111.

Moseley, F. (2015), “Introduction”, en K. Marx (ed.), Marx's Economic Manuscript of 1864-1865, Leiden, Brill, 1-44.

(2016), Money and Totality: A Macro-Monetary Interpretation of Marx's Logic in "Capital" and the End of the 'Transformation Problem', Leiden, Brill.

Munro, D. (2012), "Land and Capital". Journal of Australian Political Economy, $\mathrm{N}^{\mathrm{o}} 70,214-232$.

Murray, R. (1977), "Value and Theory of Rent", Capital \& Class, 1 (3), 100-122. Neocosmos, M. (1982), Agrarian reform and the development of capitalism in agriculture, Bradford, University of Bradford, $\mathrm{PhD}$ Thesis.

Oyhantçabal, G. y Narbondo, I. (2013), "El Agronegocio y la Expansión del Capitalismo en el Campo Uruguayo", Rebela. Revista Brasileira de Estudos Latino-Americanos, 2 (3), 409-425.

Park, J. (2014), “Land Rent Theory Revisited”, Science \& Society, 78 (1), 88-109. 
(2010), "Revisiting Marxian ground-rent theory for urban context", First International Conference in Political Economy, Crete, International Initiative for Promoting Political Economy, 1-30.

Plasencia, M. A. (1995), Renta agraria y acumulación, Buenos Aires, Programa de Investigaciones Económicas sobre Tecnología, Trabajo y Empleo, Conicet.

Ramirez, M. D. (2009), "Marx's theory of ground rent: a critical assessment", Contributions to Political Economy, 28, 71-91.

Reichelt, H. (1970), Zur logischen Struktur des Kapitalbegriffs bei Karl Marx, Freiburg, Ça ira.

Reuten, G. (2002), "Marx's Capital III, the Culmination of Capital", en M. Campbell y G. Reuten (edits.), The Culmination of Capital. Essays on Volume III of Marx's Capital, Houndmills, Basingstoke, Palgrave, 1-15. (2000), "The Interconnection of Systematic Dialectics and Historical Materialism", Historical Materialism, 7 (1), 137-165.

Ricardo, D. (1985 [1817]), Principios de economía política y tributación, México, Fondo de Cultura Económica.

Robles Baez, M. (1999), “La influencia del método 'lógico-histórico' de Engels en las interpretaciones sobre el objeto de la sección primera del tomo 1 de El capital de Marx: crítica y propuesta”, Economía Teoría y Práctica, (11), 99-123.

Rodríguez, J. (2004), "Renta, cambio tecnológico y desarrollo agropecuario: una explicación del caso argentino", VI Jornadas Nacionales de Sociología. ¿Para qué la sociología en la Argentina actual?, Buenos Aires, Carrera de Sociología, Facultad de Ciencias Sociales, Universidad de Buenos Aires.

Rosdolsky, R. (1989 [1968]), Génesis y estructura de El Capital de Marx, México, Siglo XXI.

Rubel, M. (2003 [1973]), "Plan y método de la 'Economía", en M. Rubel, Marx sin mito, Barcelona, Octaedro, 37-92.

Sartelli, E. (2008), Patrones en la ruta, Buenos Aires, Razón y Revolución.

Schmidt, A. (1973 [1972]), Historia y Estructura. Crítica del estructuralismo marxista, Madrid, Alberto Corazón Editor.

Sweezy, P. (1973 [1942]), Teoría del desarrollo capitalista, México, Fondo de Cultura Económica. 
248 ECONOMÍA TeOrÍA Y PrÁCTICA • Nueva Época, número 47, julio-diciembre 2017

Vergopoulos, K. (1977 [1974]), “El capitalistmo disforme”, en S. Amin y K. Vergopoulos, La cuestión campesina y el capitalismo, México, Editorial Nuestro Tiempo, 237.

Vlachou, A. (2002), "Nature and Value Theory", Science \& Society, 66 (2), 169201. 